\title{
Time-resolved photoelectron imaging with a femtosecond vacuum-ultraviolet light source: Dynamics in the A\#/B\#- and F\#- bands of SO2
}

\section{Journal Article}

Author(s):

Svoboda, Vít; Ram, Niraghatam B.; Rajeev, Rajendran; Wörner, Hans Jakob

Publication date:

2017-02-28

Permanent link:

https://doi.org/10.3929/ethz-a-010890580

Rights / license:

In Copyright - Non-Commercial Use Permitted

Originally published in:

The Journal of Chemical Physics 146(8), https://doi.org/10.1063/1.4976552

\section{Funding acknowledgement:}

307270 - Measuring attosecond electron dynamics in molecules (EC)

159875 - Attosecond transient-absorption spectroscopy of electronic dynamics in molecules (SNF) 


\title{
Time-resolved photoelectron imaging with a femtosecond vacuum-ultraviolet light source: dynamics in the $\tilde{\mathrm{A}} / \tilde{\mathrm{B}}-$ and $\tilde{\mathrm{F}}$-bands of $\mathrm{SO}_{2}$
}

\author{
Vít Svoboda, ${ }^{1}$ Niraghatam Bhargava Ram, ${ }^{1}$ Rajendran Rajeev, ${ }^{1}$ and Hans Jakob Wörner ${ }^{1, a)}$ \\ ETH Zurich, Laboratory of Physical Chemistry, Vladimir-Prelog-Weg 2, CH-8093 Zurich, \\ Switzerland.
}

(Dated: January 16, 2017)

Time-resolved photoelectron imaging is demonstrated using the third harmonic of a 400-nm femtosecond laser pulse as the ionization source. The resulting 133-nm pulses are combined with 266-nm pulses to study the excited-state dynamics in the $\tilde{\mathrm{A}} / \tilde{\mathrm{B}}$ - and $\tilde{\mathrm{F}}$-band regions of $\mathrm{SO}_{2}$. The photoelectron signal from the molecules excited to the $\tilde{\mathrm{A}} / \tilde{\mathrm{B}}$-band does not decay for at least several picoseconds, reflecting the population of bound states. The temporal variation of the photoelectron angular distribution (PAD) reflects the creation of a rotational wave packet in the excited state. In contrast, the photoelectron signal from molecules excited to the $\tilde{\mathrm{F}}$-band decays with a time constant of $80 \mathrm{fs}$. This time constant is attributed to the motion of the excited-state wave packet out of the ionization window. The observed time-dependent PADs are consistent with the $\tilde{\mathrm{F}}$ band corresponding to a Rydberg state of dominant s character. These results establish loworder harmonic generation as a promising tool for time-resolved photoelectron imaging of the excited-state dynamics of molecules, simultaneously giving access to low-lying electronic states, as well as Rydberg states, and avoiding the ionization of unexcited molecules.

Keywords: $\mathrm{SO}_{2}$, excited-state dynamics, time-resolved photoelectron spectroscopy, velocity-map imaging

\section{INTRODUCTION}

Time-resolved photoelectron spectroscopy (TRPES), coupled with velocity-map-imaging (VMI) techniques ${ }^{1-3}$, has become a powerful tool for investigating molecular excited-state dynamics ${ }^{4-8}$. By measuring the photoelectron velocity and angular distributions, a wealth of information can be obtained about the photoionization process and the structure and dynamics of excited states.

In the past two decades, the majority of TRPES studies have been conducted with (deep)-ultraviolet (D)UV pulses which have been broadly available from commercial laser sources. The most popular wavelength of $200 \mathrm{~nm}$ corresponds to a photon energy of $6.2 \mathrm{eV}$, which is sufficient to ionize electronically-excited states of many molecules. A variety of pump-probe studies have been performed with such sources (see e.g. Refs. ${ }^{5,7}$ and references therein). However, a photon energy of $6.2 \mathrm{eV}$ is never sufficient to ionize from the electronic ground state or very low-lying electronic states, nor is it sufficient to directly excite ground-state molecules to high-lying electronic states, in particular Rydberg states. Therefore sources of vacuum ultraviolet (VUV) radiation with femtosecond pulse duration are a highly desirable tool in the study of excited-state dynamics of molecules. The ideal light sources for such studies would combine continuous tunability from the visible into the VUV with Fourierlimited pulse durations that can be selected according to the required energy resolution or selectivity.

The discovery and intense development of highharmonic generation (HHG) has opened an attractive

\footnotetext{
a)Electronic mail: hwoerner@ethz.ch; www.atto.ethz.ch
}

path towards this ambitious goal. Most work in HHG has however concentrated on the generation of high photon energies from the vacuum ultraviolet (VUV) over the extreme ultraviolet (XUV) to the soft-X-ray domain (see e.g. Refs. ${ }^{9,10}$ and references therein). Most significant in the context of TRPES is the development of time-preserving monochromators (see e.g. Refs. ${ }^{11,12}$ and references therein), which have brought XUV-TRPES into reach ${ }^{13-15}$. While this is an extremely promising method with the potential of substantially broadening the scope of TRPES by giving access to multiple final states and element-specific core shells, XUV-TRPES suffers from the background signal contributed from the ionization of unexcited molecules. Since single-photon excitation probabilities always need to be kept low to avoid multi-photon excitation and ionization, this background constitutes a significant challenge. Time-resolved high-harmonic spectroscopy (TRHHS) turns this problem into an advantage by exploiting the emission from unexcited molecules as a coherent reference, against which the weaker signal of the photoexcited molecules is detected with phase sensitivity ${ }^{16-20}$. VUV-TRPES, in contrast, avoids the ionization of the unexcited molecules altogether, while still offering the possibility to ionize lowlying electronic states ${ }^{21}$. The other advantage of femtosecond VUV radiation is that it provides access to the ultrafast dynamics of molecular Rydberg states, which were previously only accessible through multi-photon excitation. Since multi-photon channels involving similar numbers of photons are almost impossible to discriminate against each other, single-photon-VUV excitation is a welcome solution.

In this article, we demonstrate time-resolved photoelectron imaging with a vacuum-ultraviolet source based on low-order harmonic generation in a semi-infinite gas 


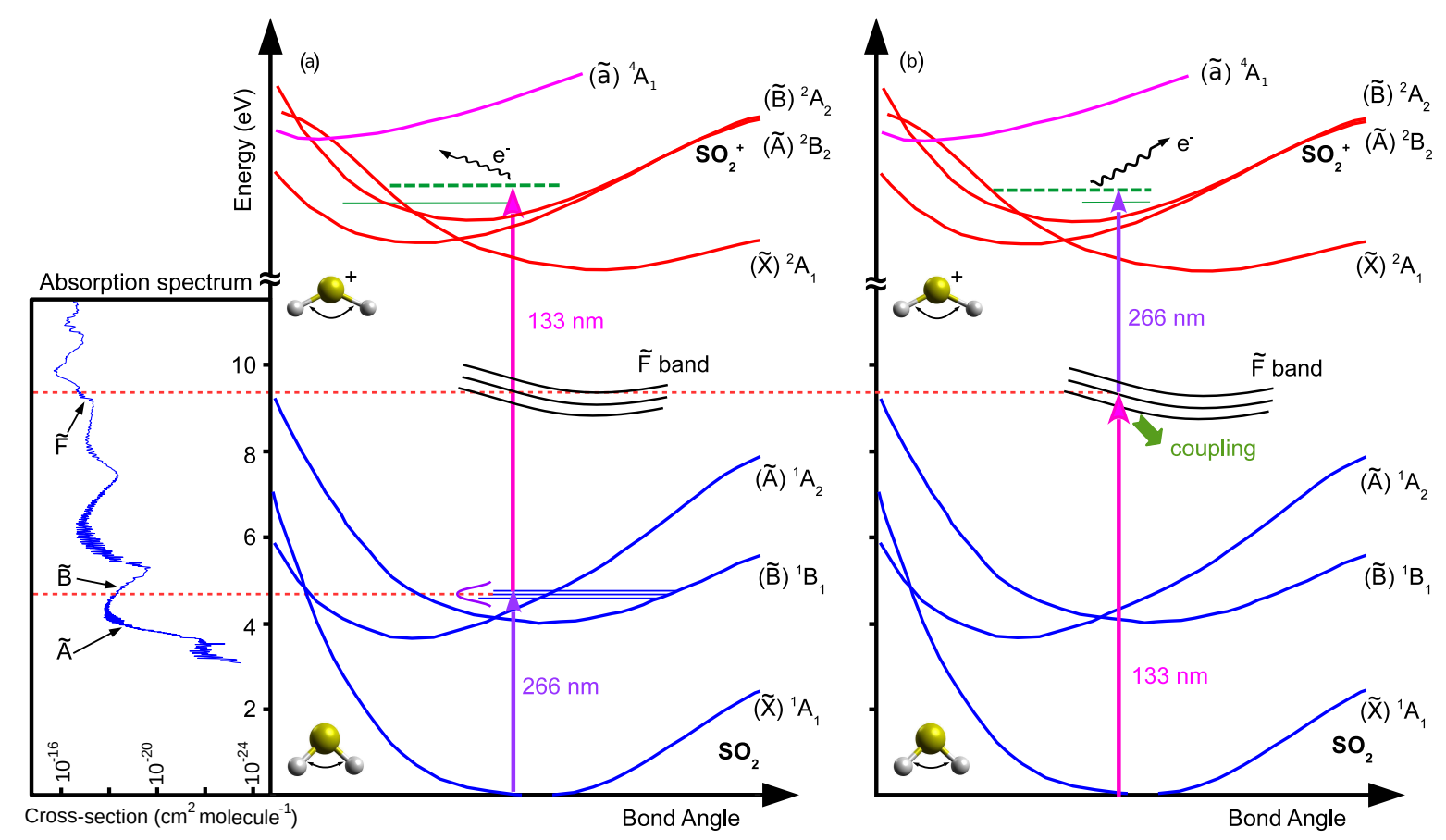

Figure 1. One-dimensional cut through potential energy surfaces of $\mathrm{SO}_{2}$ along the bond-angle coordinate from Ref. ${ }^{22}$. The diagram shows the two excitation schemes used in this work and the involved electronic states. On the left side, an absorption spectrum of $\mathrm{SO}_{2}$ is attached and the photoexcited regions are highlighted. Depending on the delay between the pulses centered at $133 \mathrm{~nm}$ and $266 \mathrm{~nm}$, two excitation paths can be distinguished: (a) at negative delays the $266 \mathrm{~nm}$ pulse pumps $\mathrm{SO}_{2}$ to the $\tilde{\mathrm{A}} / \tilde{\mathrm{B}}$ band and the subsequent $133 \mathrm{~nm}$ pulse acts as the probe, (b) at positive delays the $133 \mathrm{~nm}$ pulse excites the $\tilde{\mathrm{F}}$ band and the subsequent $266 \mathrm{~nm}$ pulse probes the $\tilde{\mathrm{F}}$-band dynamics.

cell. We study the $\mathrm{SO}_{2}$ molecule as an example to demonstrate the potential offered by this technique. We use the VUV pulses as a time-resolved probe to resolve the dynamics in the low-lying electronic states of $\mathrm{SO}_{2}$, photoexcited by UV pulses centered at $266 \mathrm{~nm}$. We then turn the role of pump and probe around and use the VUV pulses to excite the $\tilde{\mathrm{F}}$-band region of $\mathrm{SO}_{2}$ and the UV pulses to probe the dynamics by photoionization.

Small molecules, such as $\mathrm{SO}_{2}$, are good candidates for such studies because full-dimensional potential-energy surfaces are often available ${ }^{23-27}$ and, therefore, TRPES measurements can be used to directly test these calculations. Even though $\mathrm{SO}_{2}$ has been extensively studied, both experimentally ${ }^{22,28-36}$ and theoretically ${ }^{24-27,37}$, the electronic structure of $\mathrm{SO}_{2}$ in the higher energy range above $\sim 8 \mathrm{eV}$, as well as the photodynamics in all electronically-excited states are only partially understood. The first, fundamental, challenge responsible for this situation is the complexity of the network of nonadiabatic coupling between multiple close-lying electronic states, causing internal conversion and intersystem crossing on similar, femtosecond, time scales. The second challenge comes from the relatively high ionization energies of $\mathrm{SO}_{2}$, which have required the use of multiphoton excitation or ionization schemes in all previous experimental studies. This has led to the unsatisfactory situation that the number of involved photons could not be unambiguously determined in the cases where timedependent ion yields were observed, and to unresolved questions concerning the role of intermediate resonances in the case where time-dependent photoelectron spectra were measured. In the most recent experimental work devoted to the ultrafast dynamics of $\mathrm{SO}_{2}$ in its $\tilde{\mathrm{A}} / \tilde{\mathrm{B}}$ band, Wilkinson et al. ${ }^{22}$ used pulses centred around $300 \mathrm{~nm}$ to probe wave-packet dynamics between the electronically coupled $\tilde{\mathrm{B}}^{1} \mathrm{~B}_{1}$ and $\tilde{\mathrm{A}}^{1} \mathrm{~A}_{2}$ states, as well as the lower-lying triplet states. This article reported oscillatory dynamics which were attributed to a time-domain signature of the Clements bands dominating this region of the $\mathrm{SO}_{2}$ absorption spectrum. The modulation depth was observed to decrease with increasing excitation energies, from 308 to $290 \mathrm{~nm}$, but measurements at $266 \mathrm{~nm}$ were not reported. Theoretical work published as a companion article $^{27}$ showed that the observed oscillations were dominated by a resonance-enhanced $(2+1)$-ionization scheme through the $\tilde{G}$ Rydberg state. Therefore, it remained unclear whether the observed oscillations were actually reflecting the changing electronic character of the photoexcited wave packet in the $\tilde{\mathrm{A}} / \tilde{\mathrm{B}}$ states or mainly the nuclear dynamics of the wave-packet returns to the configuration-space window enabling efficient resonanceenhanced $(2+1)$-ionization. This example illustrates the general necessity of femtosecond VUV light sources to disentangle molecular excited-state dynamics. 
In the present work, we focus our attention on the ultrafast dynamics occurring upon photoexcitation to the $\tilde{\mathrm{A}} / \tilde{\mathrm{B}}$ and $\tilde{\mathrm{F}}$ states. To unambiguously reveal the dynamics of the $\tilde{\mathrm{A}} / \tilde{\mathrm{B}}$ and $\tilde{\mathrm{F}}$ states, we used a two-color pump-probe scheme in the single-photon-absorption regime to avoid the complication induced by multiphoton processes in both excitation and ionization. The pump-probe scheme is depicted in Figure 1. By recording electron-momentum images we were able to elucidate the dynamics after photoexcitation in the region of the $\tilde{\mathrm{A}} / \tilde{\mathrm{B}}$ and $\tilde{\mathrm{F}}$ bands. The excitation by a $266-\mathrm{nm}$ pulse initiates a nuclear wavepacket in the optically allowed $\tilde{\mathrm{B}}^{1} \mathrm{~B}_{1}$ state, which undergoes rapid internal conversion to the dark $\tilde{\mathrm{A}}^{1} \mathrm{~A}_{2}$ state. This situation is depicted in Figure 1 panel (a). As can be seen in the absorption spectra of Figure 1, this energy region is characterized by a high density of vibronic states forming a quasi-continuum, which supports complex wave-packet dynamics. Wave-packet propagation on potential-energy surfaces from high-level ab initio calculations nevertheless predict a periodic evolution of the diabatic populations in the $\tilde{\mathrm{B}}^{1} \mathrm{~B}_{1}$ and $\tilde{\mathrm{A}}^{1} \mathrm{~A}_{2}$ states with a period of $\sim 120 \mathrm{fs}$ and a contrast of up to $25 \%^{38}$.

Excitation at $133 \mathrm{~nm}$ allows us to explore the dynamics of the $\tilde{\mathrm{F}}$ band, which to the best of our knowledge, have not been studied by photoelectron spectroscopy before. The excitation by $133-\mathrm{nm}$ pulses provides direct singlephoton access to the $\tilde{\mathrm{F}}$ band, where the onset of Rydberg series are predicted ${ }^{37}$. Previous experimental work of Zhang et al. ${ }^{28}$, studying the time-dependent ion yields after multi-photon excitation to the $\tilde{\mathrm{F}}$ band, suggested dissociative dynamics to take place on the time scale of a few-picosecond time scale.

The paper is organized as follows: Section II describes the experimental setup and summarizes the typical experimental conditions used for recording electron images, followed by the description of the data analysis. Section III presents the results and the discussion of timeresolved ion and electron yields, as well as those of timeand angle-resolved photoelectron spectra. Each of these results is discussed according to the selected pump-probe scheme and compared with the relevant theoretical and experimental work. The results presented in this paper are summarized in section IV.

\section{EXPERIMENT}

All data presented in this article have been recorded using a two-color pump-probe scheme utilizing low-orderharmonic generation (LOHG) in a semi-infinite gas cell, and a velocity-map-imaging spectrometer (VMIS) ${ }^{39,40}$. The setup is schematically depicted in Figure 2.

We used $1.5 \mathrm{~mJ}$ pulses with 35 fs duration from a Ti:Sa regenerative amplifier operating at $1 \mathrm{kHz}$ and used a 70:30 beam splitter. The transmitted beam was delayed with respect to the reflected beam using a motorized delay stage. After the delay stage, the transmitted beam was frequency doubled using a $300 \mu \mathrm{m}$ thick $\beta$ -

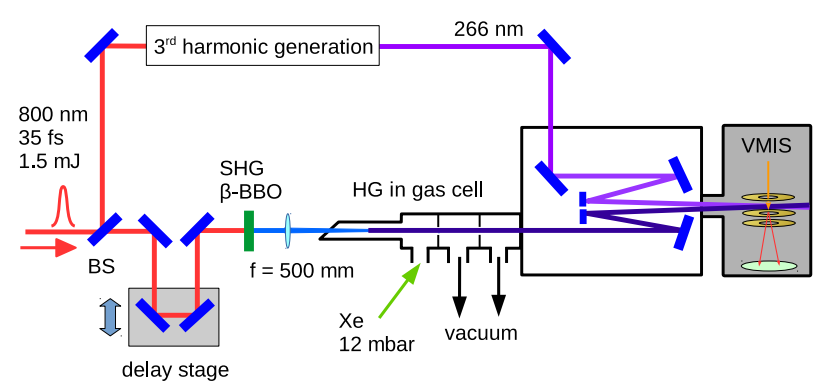

Figure 2. Schematic diagram of the experimental setup consisting of a two-color pump-probe interferometer and velocitymap-imaging spectrometer (VMIS).

barium borate crystal (BBO) to obtain a $400 \mathrm{~nm}$ beam. The second-harmonic beam was separated from the fundamental by reflections on two dichroic mirrors and then focused by a $\mathrm{f}=500 \mathrm{~mm}$ lens into a semi-infinite gas cell filled with 12 mbar of Xe. The pressure of the generation gas was chosen such that the third harmonic of $400 \mathrm{~nm}$ $(133 \mathrm{~nm})$ was optimized. Under these conditions, no photoelectrons originating from the fifth harmonic $(80 \mathrm{~nm})$ or higher orders were observed. The reflected beam, after the beam-splitter, was used for the third-harmonic generation giving an output beam centered around $266 \mathrm{~nm}$.

Both beams were guided into a vacuum chamber where they were focused non-collinearly (with a crossing angle of less than $1^{\circ}$ ), by two spherical mirrors $(\mathrm{f}=0.5 \mathrm{~m}$ and $\mathrm{f}=1 \mathrm{~m}$ for $266 \mathrm{~nm}$ ). Mirrors for the $266 \mathrm{~nm}$ beam were coated in such a way to transmit the residual $800 \mathrm{~nm}$ and $400 \mathrm{~nm}$ beams from the third-harmonic-generation process. The mirrors were fixed on motorized mounts allowing for fine adjustment of the spatial overlap under vacuum. The beams were focused through a small slit $\left(15 \times 5 \mathrm{~mm}^{2}\right)$ separating the mirror chamber from the VMI spectrometer for optimal differential pumping.

A $1 \%$ mixture of $\mathrm{SO}_{2}$ (PanGas $99.995 \%$ purity) in $\mathrm{Ne}$ was expanded through a pulsed valve (Even-Lavie, orifice $150 \mu \mathrm{m}$ ) working at a repetition rate of $1 \mathrm{kHz}$ using a backing pressure of about 2 bar to form a supersonic molecular beam. The gas jet was skimmed by a $500 \mu \mathrm{m}$ skimmer and propagated $15 \mathrm{~cm}$ downstream to reach the interaction region where it was ionized by the combined action of the laser beams. A cross-correlation of 102 fs (FWHM) was determined by two-color ionization of Xe (see Figure 3). The cross-correlation function was taken into account during data analysis as an instrument response function. Ejected electrons were imaged using electrostatic lenses fulfilling VMI conditions, and a microchannel-plate (MCP) detector in a Chevron configuration. Electron images were recorded using a ratio of electric potentials on the repeller (R) and extractor (E) electrodes of $V_{R}: V_{E}=5: 4$.

Time-resolved photoelectron spectra (TRPES) were recorded for pump-probe delays between -1 and $+1 \mathrm{ps}$ in steps of $20 \mathrm{fs}$. The negative delays refer to the situation where the $266-\mathrm{nm}$ pulse arrives before the $133-\mathrm{nm}$ 


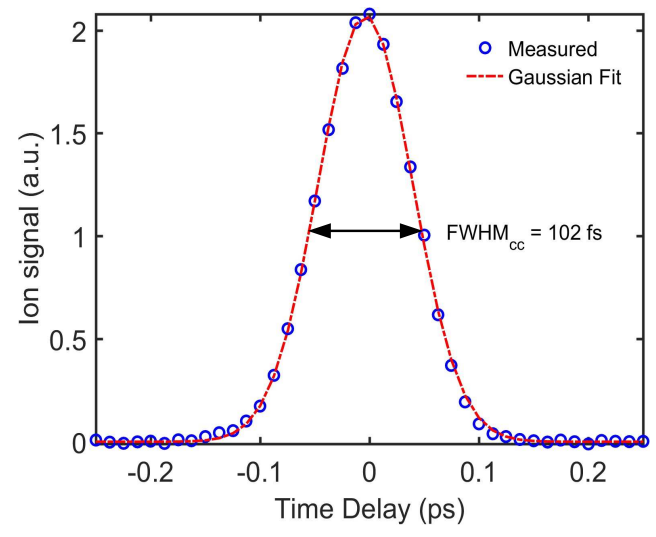

Figure 3. Experimentally determined non-resonant crosscorrelation in Xe using 266-nm and 133-nm pulses. A singleGaussian fit was used to determine the cross-correlation FWHM $_{\mathrm{cc}}$.

pulse. The TRPES spectrum was constructed from measured data as follows. For each time step, three photoelectron images were recorded: one two-color image and two one-color images (where one of the two beams was blocked), respectively. One-color data were then subtracted from the two-color data to obtain backgroundfree data containing only contributions from the twocolor beams. These images were inverted using a pBasex inversion method ${ }^{41} .34$ scans were performed serially and the total yields were integrated for each delay step. Finally, the TRPES spectrum was constructed from averaged images by numerical integration. Calibration of the energy axis was done using Xe measurements recorded under the same VMI conditions.

\section{RESULTS AND DISCUSSION}

\section{A. Time-dependent ion yields}

We first discuss the time-resolved ion-yield measurements. The time dependence of the $\mathrm{SO}_{2}^{+}$yield is summarized in Figure 4. The negative-delay side shows a decay of the ion signal within the cross-correlation time to a signal level that remains constant for, at least, several picoseconds. This is explained by the fact that the 266-nm pulse excites $\mathrm{SO}_{2}$ to the bound $\tilde{\mathrm{B}}^{1} \mathrm{~B}_{1}$ state which undergoes rapid internal conversion to the $\tilde{\mathrm{A}}^{1} \mathrm{~A}_{2}$ state and intersystem crossing to three lowerlying triplet states ${ }^{22,26}$. All of these five states are bound. The population dynamics following excitation at $266 \mathrm{~nm}$ has been calculated following the methods described in Ref. ${ }^{27}$. This calculation, as mentioned in the introduction, predicts population transfer between the diabatic $\tilde{\mathrm{B}}^{1} \mathrm{~B}_{1}$ and $\tilde{\mathrm{A}}^{1} \mathrm{~A}_{2}$ states with a period of $\sim 120 \mathrm{fs}^{38}$. This period would clearly be resolved under the conditions of our experiment. Its absence from the measured ion yield therefore shows that one-photon ionization at 133 $\mathrm{nm}$ is not sensitive to the dynamics of internal conversion taking place at the conical intersection between the $\tilde{\mathrm{B}}^{1} \mathrm{~B}_{1}$ and $\tilde{\mathrm{A}}^{1} \mathrm{~A}_{2}$ states.

The positive-delay side of Fig. 4 shows an exponential decay to zero signal level, which is well represented by a single-exponential function with a time constant of $80 \pm 27$ fs convoluted with the cross-correlation function. Photoexcitation at $133 \mathrm{~nm}$ populates the socalled $\tilde{\mathrm{F}}$-band of $\mathrm{SO}_{2}$ at $9.3 \mathrm{eV}$ which has been assigned to the lowest-lying Rydberg state converging to the $\tilde{\mathrm{X}}^{+2} \mathrm{~A}_{1}$ state of the ion. Calculations locate the lowestlying singlet Rydberg state of dominant s-character in this energy region ${ }^{37}$. Since this Rydberg state is most probably bound, just as the $\tilde{\mathrm{X}}^{+2} \mathrm{~A}_{1}$ state to which it converges, the rapid observed decay is most likely to be caused by predissociation induced by coupling to a repulsive Rydberg or valence state. This conclusion is supported by the time-resolved photoelectron signals discussed below.

In contrast to photoexcitation of $\mathrm{SO}_{2}$ around $266 \mathrm{~nm}$, which has been studied by different methods, only a single time-resolved study of excited-state dynamics following photoexcitation to the $9.3 \mathrm{eV}$ energy range has been reported. It is a multiphoton-excitation femtosecond ionyield measurement by Zhang and co-workers ${ }^{28}$. These authors investigated time-resolved ion yields of the parent ion $\left(\mathrm{SO}_{2}^{+}\right)$and the fragment ions $\left(\mathrm{SO}^{+}, \mathrm{S}^{+}\right.$, and $\left.\mathrm{O}^{+}\right)$. Biexponential decays were fitted to all transients. For $\mathrm{SO}_{2}^{+}$, a fast decay component of $280 \mathrm{fs}$ and a slow decay component of 2.95 ps was obtained. The fast component was attributed to a coupling between the $\tilde{\mathrm{F}}$ and $\tilde{\mathrm{E}}$ states, whereas the slow component was assigned to direct dissociation from the $\tilde{\mathrm{F}}$ state. In our time-resolved $\mathrm{SO}_{2}^{+}$ion yield, there is no evidence for a long-lived (ps) component and our short time constant $(80 \pm 27 \mathrm{fs})$ is much shorter than that of Zhang et al..

This discrepancy is probably explained by the higher pump intensity used in Ref. ${ }^{28}$. It is indeed very likely that dynamics in low-lying excited states of $\mathrm{SO}_{2}^{+}$contribute to the results in Ref. ${ }^{28}$, because it is impossible to excite an appreciable fraction of the molecules with 2 photons at $266 \mathrm{~nm}$ without absorbing a third photon, which would leave $\mathrm{SO}_{2}^{+}$in any of its $\tilde{\mathrm{X}}^{+2} \mathrm{~A}_{1}, \tilde{\mathrm{A}}^{+2} \mathrm{~B}_{2}$ or $\tilde{\mathrm{B}}^{+2} \mathrm{~A}_{2}$ states. A time-resolved ion-yield measurement does indeed not distinguish between dynamics taking place in the neutral as opposed to the ionic manifolds of electronic states. Our experiments, in contrast, are carried out in the regime of single-photon processes for both pump and probe pulses. Therefore, the decay of the $\mathrm{SO}_{2}^{+}$signal in our measurements can safely be assigned to dynamics initiating in the $\tilde{\mathrm{F}}$ state. This conclusion is further corroborated by time-resolved photoelectron measurements described in the next section. 


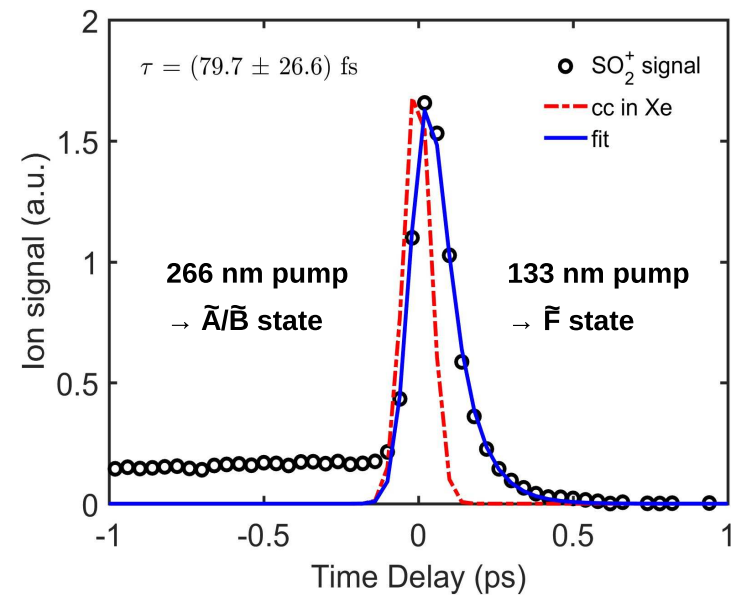

Figure 4. Time-dependent ion yield for $\mathrm{SO}_{2}^{+}$. The red dashdotted line corresponds to the cross-correlation signal determined in $\mathrm{Xe}$ (see Figure 3). The blue line is a monoexponential decay convoluted with the cross-correlation function.

\section{B. Time-resolved photoelectron spectra}

The TRPES spectra are shown in Figure 5 as a function of the photoelectron energy and the time delay between the pump and probe pulses. A first inspection of the $2 \mathrm{D}$ map reveals an intense feature around time zero, which corresponds to the absorption of one photon from each pulse. At negative delays, a broad feature covering energies from $0 \mathrm{eV}$ up to $\sim 0.6 \mathrm{eV}$ is observed, which is about ten times weaker than the signal at time zero. At positive delays, a rapidly decaying feature can be observed around $1.5 \mathrm{eV}$. A much weaker signal is observed at positive delays between $0 \mathrm{eV}$ and $\sim 0.3 \mathrm{eV}$.

\section{Detailed analysis of the TRPES spectrum}

In this section we discuss the TRPES data in more detail. To simplify the discussion, we subdivide this section into two parts: (i) negative-delay and (ii) positive-delay side of the TRPES spectrograms. The discussion follows the energy diagram presented in Fig. 1, which shows the excitation schemes and the electronic states prepared by photoexcitation.

\section{Negative delays}

On the negative-delay side, the 266-nm pulse acts as the pump and a subsequent 133-nm pulse is the probe (see excitation path (a) in Figure 1). According to the theoretical calculations ${ }^{22,37,42}$, the pump pulse at this particular wavelength photoexcites molecules from the $\tilde{\mathrm{X}}^{1} \mathrm{~A}_{1}$ electronic ground state to the optically bright $\tilde{\mathrm{B}}^{1} \mathrm{~B}_{1}$ state. In this excitation region, the absorption spectrum is more complex compared with the well- studied $\tilde{\mathrm{A}}$ band (containing the Clements bands). The complexity of the absorption spectrum at these higher energies arises from non-adiabatic coupling between the ${ }^{1} \mathrm{~B}_{1}$ and ${ }^{1} \mathrm{~A}_{2}$ electronic states, spin-orbit coupling to several lower-lying triplet states ${ }^{24,25}$, and from the fact that the $\tilde{\mathrm{B}}^{1} \mathrm{~B}_{1}$ state is coupled to the high-lying vibrational levels of the $\tilde{\mathrm{X}}^{1} \mathrm{~A}_{1}$ ground state via Renner-Teller coupling ${ }^{42}$.

In total, the pump and probe pulses deposit $13.95 \mathrm{eV}$ of energy into the molecule. This energy is sufficient to populate three cationic states, namely $\tilde{\mathrm{X}}^{+}{ }^{2} \mathrm{~A}_{1}$, $\tilde{\mathrm{A}}^{+}{ }^{2} \mathrm{~B}_{2}$, and $\tilde{\mathrm{B}}^{+}{ }^{2} \mathrm{~A}_{2}\left(I_{\mathrm{p}}=12.35,12.99\right.$, and $13.34 \mathrm{eV}$, respectively $\left.{ }^{31,43}\right)$. If ionization was taking place to the vibronic ground state of each of these three states, one could expect three photoelectron bands centered around $1.61,0.97$, and $0.62 \mathrm{eV}$, respectively.

The experimental spectra show an intense photoelectron band appearing at 1.5-1.6 eV with a long tail extending to low kinetic energies, which decays within the cross-correlation time towards the negative-delay side. A magnified representation of the low-energy region (inset of Fig. 5) shows a weaker broad photoelectron band covering kinetic energies up to $\sim 0.6 \mathrm{eV}$, which does not decay within the measured time interval. The observed kinetic energy is substantially lower than the maximal available photoelectron kinetic energy of $1.6 \mathrm{eV}$. Hence, ionization is taking place to vibrationally-excited states of $\mathrm{SO}_{2}^{+}$.

Koopman's correlations predict dominant ionization from the $\tilde{\mathrm{B}}^{1} \mathrm{~B}_{1}$ state of $\mathrm{SO}_{2}$ to the $\tilde{\mathrm{X}}^{+2} \mathrm{~A}_{1}$ state of $\mathrm{SO}_{2}^{+}$ and from the $\tilde{\mathrm{A}}^{1} \mathrm{~A}_{2}$ state to the $\tilde{\mathrm{A}}^{+2} \mathrm{~B}_{2}$ state. Since we observe a single broad band at very low energies at negative delays, we conclude that ionization takes place to vibrationally excited states of the $\tilde{\mathrm{X}}^{+2} \mathrm{~A}_{1}$ and $\tilde{\mathrm{A}}^{+2} \mathrm{~B}_{2}$ states. This conclusion is also consistent with the fact that the potential energy surfaces of the $\tilde{\mathrm{X}}^{+2} \mathrm{~A}_{1}$ and $\tilde{\mathrm{A}}^{+2} \mathrm{~B}_{2}$ states of $\mathrm{SO}_{2}^{+}$are almost parallel to those of the $\tilde{\mathrm{B}}^{1} \mathrm{~B}_{1}$ and $\tilde{\mathrm{A}}^{1} \mathrm{~A}_{2}$ states of $\mathrm{SO}_{2}$, respectively, as shown in Fig. 1, such that Franck-Condon factors will be close to diagonal.

As in the case of the time-resolved photoion yield, shown in Fig. 4, the time-resolved photoelectron yield does also not show any simple temporal variation within the first picosecond. The observation of a nearly timeindependent photoelectron spectrum is quite remarkable given the fact that the excited-state wave packet is known to perform large-amplitude motion in the coupled $\tilde{\mathrm{A}} / \tilde{\mathrm{B}}$ states. This observation can however be interpreted to be a consequence of the very similar shapes of the $\tilde{\mathrm{B}}^{1} \mathrm{~B}_{1}$ and $\tilde{\mathrm{X}}^{+2} \mathrm{~A}_{1}$ states on one hand, and the $\tilde{\mathrm{A}}^{1} \mathrm{~A}_{2}$ and $\tilde{\mathrm{A}}^{+2} \mathrm{~B}_{2}$ states on the other hand, which are both related by the removal of the most weakly bound electron out of the $b_{1}$ orbital. It is moreover known from previous work ${ }^{24}$ that intersystem crossing is also taking place on the observed time scale and that it dominantly populates the $(1)^{3} \mathrm{~B}_{2}$ state. The potential-energy surface of this state again has a very similar shape to that of the $\tilde{\mathrm{B}}^{+2} \mathrm{~A}_{2}$ state, to which it is connected by ionization from the outermost $b_{1}$ orbital. It is therefore again understandable that the intersystem crossing does not 


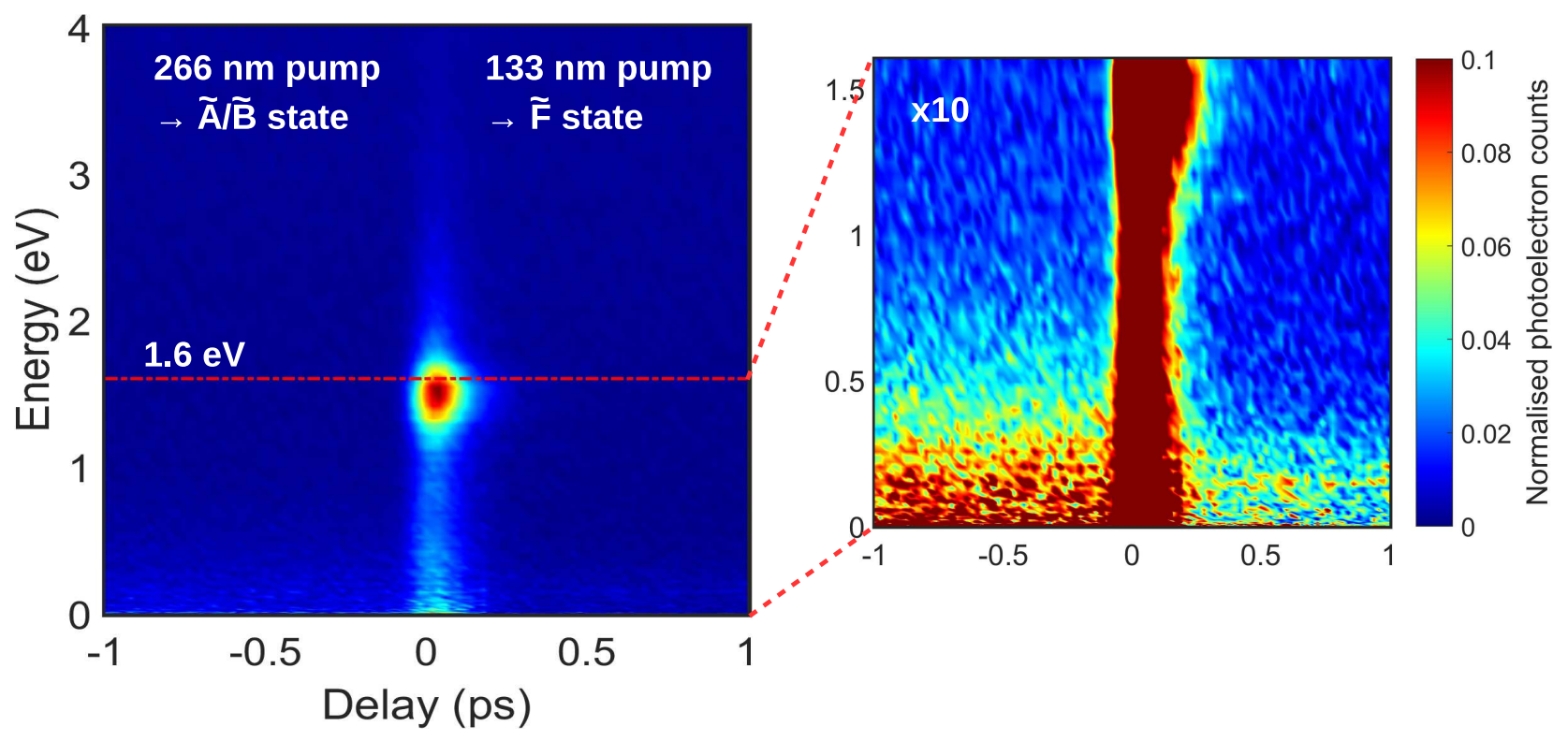

Figure 5. (Left panel) Time-resolved photoelectron spectrum (TRPES) of $\mathrm{SO}_{2}$ molecules, ionized by a combination of femtosecond pulses centered at $266 \mathrm{~nm}$ and $133 \mathrm{~nm}$. The time delay between the pulses was varied from -1 to $1 \mathrm{ps}$ with steps of $20 \mathrm{fs}$. Interpretation of the TRPES spectrum, as well as the information how the spectrum was constructed from measured data can be found in the main text. (Right panel) A magnification of low-energy photoelectron band shows a broad signal at long negative and positive delays.

strongly manifest itself in the presented data. Therefore our experiment shows that TRPES, as implemented in the present work, is neither sensitive to the dynamics of internal conversion taking place at the conical intersection between the $\tilde{\mathrm{B}}^{1} \mathrm{~B}_{1}$ and $\tilde{\mathrm{A}}^{1} \mathrm{~A}_{2}$ states of $\mathrm{SO}_{2}$, nor to the intersystem crossing that has been predicted to take place on a sub-picosecond time scale.

\section{Positive delays}

On the positive-delay side, the $133-n m$ pulse acts as the pump pulse whereas the time-delayed 266-nm pulse is the probe (excitation path (b) in Figure 1). The excitation at $133 \mathrm{~nm}$ populates the $\tilde{\mathrm{F}}$-state manifold, which starts at $9.25 \mathrm{eV}^{37}$. This spectral region contains the onset of Rydberg series converging to the three lowest-lying electronic states of $\mathrm{SO}_{2}^{+}$. The lowest Rydberg state (3stype Rydberg state converging to the $\tilde{\mathrm{X}}^{+2} \mathrm{~A}_{1}$ state of $\mathrm{SO}_{2}^{+}$) is predicted to lie at $\sim 9.3 \mathrm{eV}$.

The measured TRPES spectra are dominated by a single band centered at $1.51 \mathrm{eV}$. In contrast to the negativedelay side, this band decays more slowly than the crosscorrelation time. Since this kinetic energy is close to the maximally-allowed one, this band can be assigned to a photoionizing transition to the vibronic ground state of the $\tilde{\mathrm{X}}^{+2} \mathrm{~A}_{1}$ state of $\mathrm{SO}_{2}^{+}$. This fact supports the assignment of the populated electronic state to a Rydberg state converging to the $\tilde{\mathrm{X}}^{+2} \mathrm{~A}_{1}$ electronic ground state of $\mathrm{SO}_{2}^{+}$, because the photoionization of Rydberg states to the cationic state to which they converge is usually asso- ciated with diagonal Franck-Condon factors. As shown in more detail in the next section and Fig. 7b, the entire photoelectron band extending from 0 to $\sim 2 \mathrm{eV}$ decays within the cross-correlation time towards negative delays and with the same time constant towards positive delays and is therefore attributed to the same 3s-type Rydberg state.

We first discuss the fast decay of the photoelectron band centered around $1.51 \mathrm{eV}$ (Figure 5). Both monoexponential (Eq. (1)) and biexponential (Eq. (2)) functions convoluted with the cross-correlation function have been fitted to this data. The fit expressions are ${ }^{44}$

$$
\begin{gathered}
S=A \cdot \exp \left(\left(\frac{\sigma}{2 \tau}\right)^{2}-\frac{t-t_{0}}{\tau}\right) \cdot\left(1-\operatorname{erf}\left(\frac{\sigma}{2 \tau}-\frac{t-t_{0}}{\sigma}\right)\right) \\
S=A_{1} \cdot \exp \left(\left(\frac{\sigma}{2 \tau_{1}}\right)^{2}-\frac{t-t_{0}}{\tau_{1}}\right) \cdot\left(1-\operatorname{erf}\left(\frac{\sigma}{2 \tau_{1}}-\frac{t-t_{0}}{\sigma}\right)\right) \\
+A_{2} \cdot \exp \left(\left(\frac{\sigma}{2 \tau_{2}}\right)^{2}-\frac{t-t_{0}}{\tau_{2}}\right) \cdot\left(1-\operatorname{erf}\left(\frac{\sigma}{2 \tau_{2}}-\frac{t-t_{0}}{\sigma}\right)\right),
\end{gathered}
$$

where Eq. (1) and Eq. (2) correspond to mono- and bi-exponential fits, respectively. Parameters $A_{i}$ are amplitudes, $\tau_{i}$ are decay constants, $t-t_{0}$ is the time delay between pump and probe pulses and $\sigma$ is the width of the cross-correlation function $\left(\sigma=\frac{\mathrm{FWHM}_{c c}}{2 \sqrt{\ln 2}} \approx 61 \mathrm{fs}\right)$.

The results of the fitting procedure are summarized in Figure 6. We compare the monoexponential and biexponential fits to estimate the contributions leading to the measured signal. The monoexponential fit gives a decay constant of $\tau=77 \pm 7 \mathrm{fs}$. In the case of the biexponential 

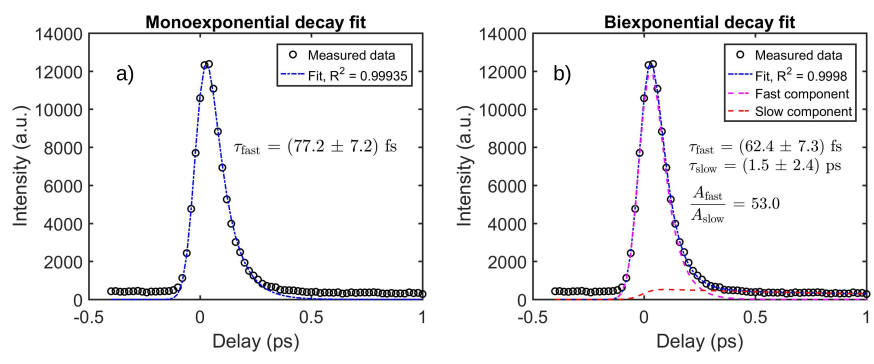

Figure 6. (a) Monoexponential and (b) biexponential fit of the temporal profile of the total photoelectron signal corresponding to the full width at half maximum of the band at $1.51 \mathrm{eV}$. For more details see the main text.

fit we obtained an amplitude ratio of $A_{\text {fast }} / A_{\text {slow }}=53$ between the fast and slow components, respectively. This ratio suggests that the slow component is almost negligible. Thus, we conclude that our experiment is mainly sensitive to a rapidly decaying component with a time constant of $\sim 80 \mathrm{fs}$. This conclusion agrees well with the decay constant derived from the $\mathrm{SO}_{2}^{+}$ion trace (see section III A).

\section{Time- and angle-resolved photoelectron spectra}

We now turn to the observation of angle-resolved photoelectron spectra and determine the time-dependent photoelectron angular distributions. For the case of linearly polarized pump and probe pulses, the intensity of each being kept in the one-photon-absorption regime, the laboratory-frame distribution of photoelectrons can be expressed as a finite series expansion in Legendre polynomials $^{6,7}$

$$
\frac{\mathrm{d} \sigma(t)}{\mathrm{d} \Omega}=\frac{\sigma(t)}{4 \pi}\left[1+\beta_{2}(t) P_{2}(\cos \theta)+\beta_{4}(t) P_{4}(\cos \theta)\right],
$$

where $\sigma(t)$ denotes the time-dependent photoionization cross section, $\theta$ the polar angle between the polarization axis of the laser pulses and the emission direction of the electron and $P_{L}$ represents the Legendre polynomial of order $L$.

Figure 7 a shows the asymmetry parameter $\beta_{2}(t, E)$ as a function of the delay $t$ and the photoelectron kinetic energy $E$. Panels b and $\mathrm{c}$ show the time-dependent asymmetry parameters $\beta_{2}(t)$ and $\beta_{4}(t)$ (blue/green lines), following integration over the areas indicated in panel a, together with the angle-integrated photoelectron signal (red dots/line).

Figure $7 \mathrm{~b}$ focuses on the low-kinetic-energy data obtained by integrating the signal in the range of $0-0.5 \mathrm{eV}$. On the negative-delay side the $\beta_{2}$ value is found to decay from $\sim 0.35$ during overlap of the pump and probe pulses to $\sim 0.2$. A weak local minimum can be discerned around delays of $0.3 \mathrm{ps}$. This time dependence is reminiscent of the behavior of rotational anisotropies following singlephoton excitation that were observed in Refs. ${ }^{45,46}$. The

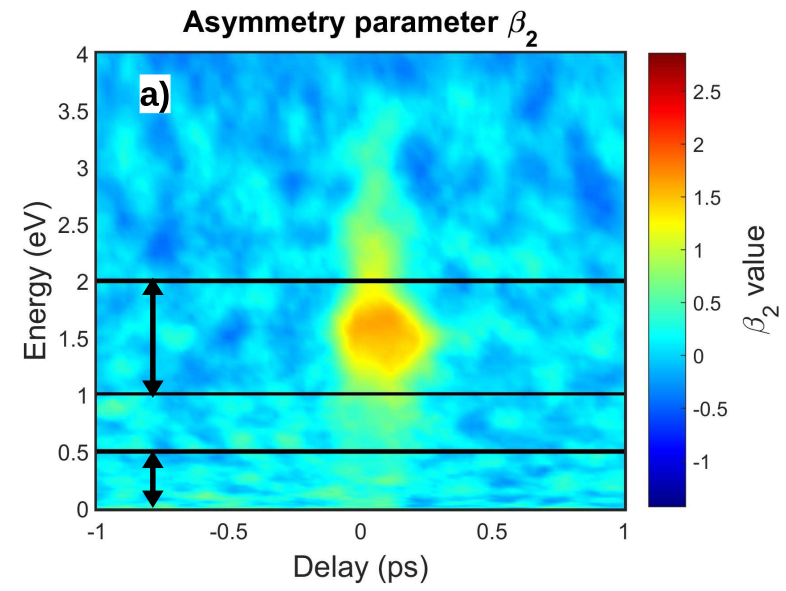

b)

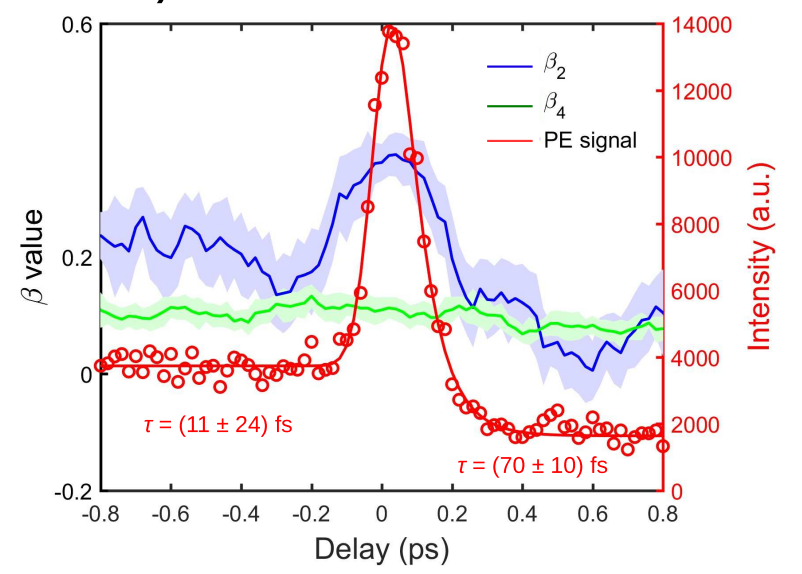

c)

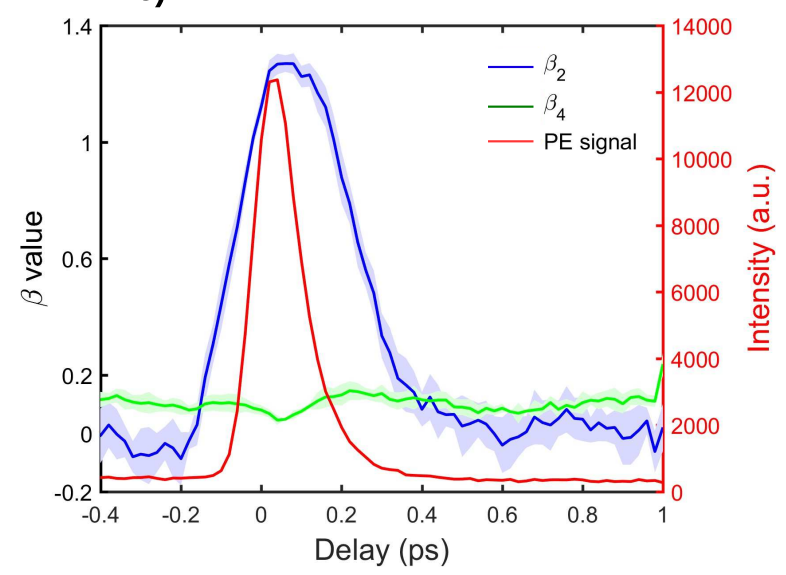

Figure 7. a) Asymmetry parameter $\beta_{2}$ as a function of the photoelectron kinetic energy $E$ and the delay $t$, b) asymmetry parameter $\beta_{2}$ and $\beta_{4}$ (blue/green lines) and photoelectron signal (red line), all obtained following integration over the interval $[0 ; 0.5] \mathrm{eV}$ in panel (a). The shaded areas represent one standard deviation. c) same as (b) following integration over the range $[1 ; 2] \mathrm{eV}$ in panel (a).

time dependence of the $\beta_{2}$ parameter is indeed markedly different from that of the angle-integrated photoelectron signal. Its decay is clearly non exponential and its asymptotic value differs significantly from zero. All of these 
observations are consistent with the expected signatures of rotational anisotropy following excitation of a bound state, as we further discuss below.

Figure $7 \mathrm{c}$ shows the positive-delay data, integrated over the range of kinetic energies from 1 to $2 \mathrm{eV}$, which reflects the dynamics following one-photon excitation of the $\tilde{\mathrm{F}}$ band. In this case, a much higher anisotropy parameter $\beta_{2} \approx 1.3$ is reached during overlap of the pump and probe pulses. This value is consistent with the population of an s-type Rydberg state because photoionization from a purely atomic s orbital would result in a pure pwave continuum, which corresponds to $\beta_{2}=2$. The $\beta_{2}$ parameter decays on a much slower time scale than the photoelectron signal. Its decay again shows a clearly nonexponential behavior, but it decays to zero within the accuracy of our measurement. This behavior indicates a qualitatively different type of dynamics compared to that of the $\tilde{\mathrm{A}} / \tilde{\mathrm{B}}$ band.
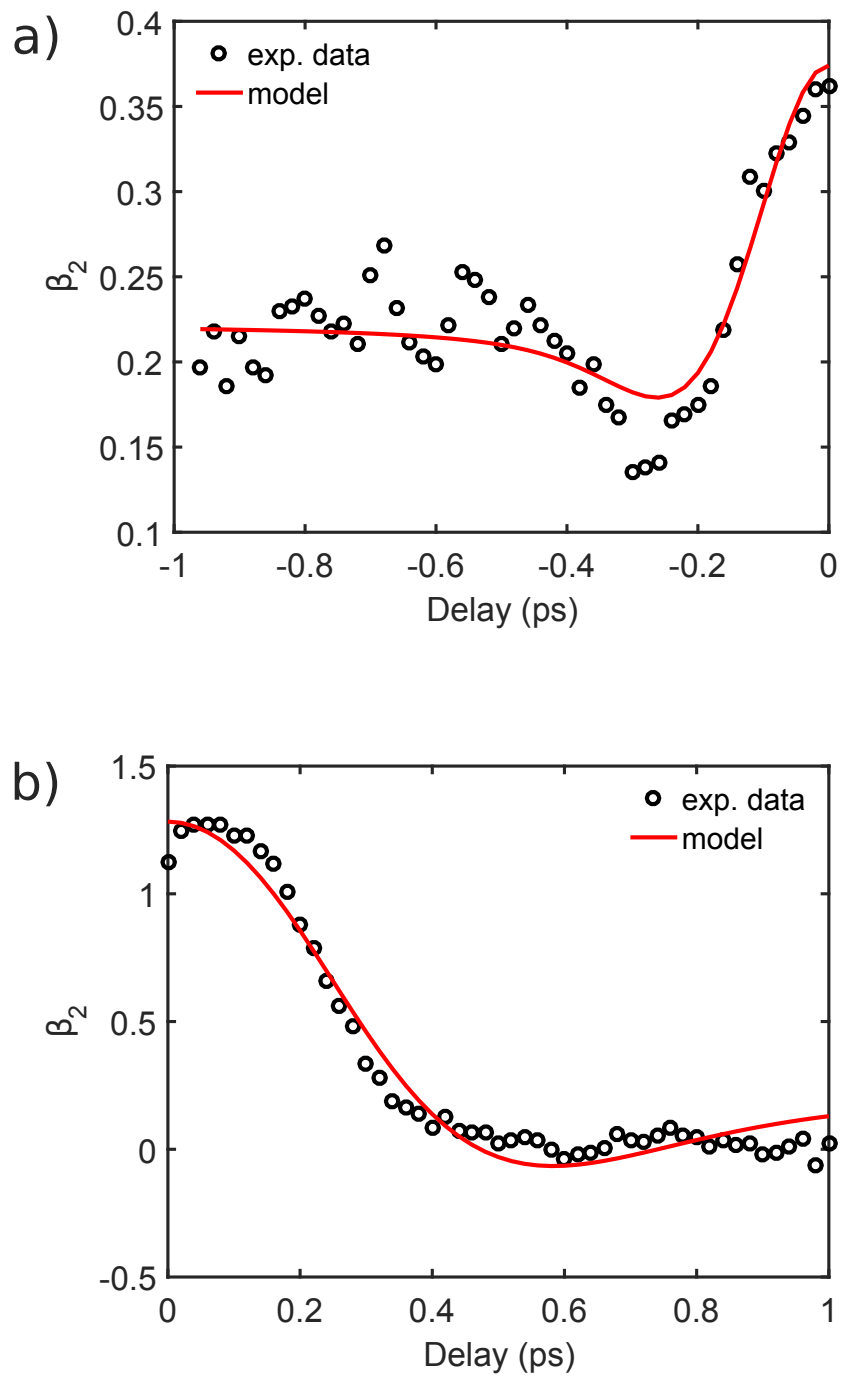

Figure 8. a) Time-dependent asymmetry parameter $\beta_{2}(t)$ from Fig. $7 \mathrm{~b}$ and model discussed in the text $\mathrm{b}$ ) same as (a) with data from Fig. 7c.
We now compare these observations of time-dependent asymmetry parameters with a recently derived analytical model of rotational anisotropies of symmetric-top molecules ${ }^{47}$. Although $\mathrm{SO}_{2}$ is an asymmetric top, its rotational constants $B$ and $C$ in the optically accessible states are sufficiently close to being equal to justify a symmetric-top approximation (see Table I). The timedependent asymmetry parameters can in general be expressed as follows:

$$
\beta_{l, q}(t)=\sum_{i=0}^{n} A_{2 i, q}(t) B_{l, 2 i, q}(t)
$$

where $A_{2 i, q}(t)$ are time-dependent axis-distribution moments defined for the case where cylindrical symmetry is preserved and $B_{l, 2 i, q}(t)$ describe the additional effect of vibrational and/or electronic dynamics on the time dependence of the asymmetry parameters.

In the case where a single vibrational state of a bound electronic state is prepared by one-photon excitation, the time-dependent axis-distribution moments $A_{2 i, q}(t)$ can easily be calculated numerically and the coefficients $B_{l, 2 i, q}(t)$ are time independent. In such cases, additionally assuming single-photon ionization, the asymmetry parameter $\beta_{2}(t)$ can be represented as

$$
\beta_{2,0}(t)=A_{0,0}(t) B_{2,0,0}+A_{2,0}(t) B_{2,2,0} .
$$

Although this case does not exactly apply to either of the two types of dynamics studied in this work, the comparison between the experimental results and this model provides additional insight into the observed dynamics. The time-dependent axis distribution moments for the $\tilde{\mathrm{A}} / \tilde{\mathrm{B}}$ dynamics have been calculated on the basis of the average of the rotational constants of the $\tilde{\mathrm{A}}$ and $\tilde{\mathrm{B}}$ states because calculations show a rapid spreading of the nuclear wave packet over both electronic states. In the case of the $\tilde{\mathrm{F}}$ Rydberg state, the rotational constants of the electronic ground state of $\mathrm{SO}_{2}^{+}$have been used, because Rydberg states have equilibrium geometries that closely resemble those of the cationic state to which they converge. All rotational constants that were used are given in Table I. The coefficients $B_{l, 2 i, q}$ were treated as timeindependent parameters in an error-weighted non-linear least-squares fitting procedure. The obtained values are $B_{2,0,0}=0.67$ and $B_{2,2,0}=-0.057$ in the case of the $\tilde{\mathrm{A}} / \tilde{\mathrm{B}}$ dynamics and $B_{2,0,0}=1.60$ and $B_{2,2,0}=-1.16$ in the case of the $\tilde{\mathrm{F}}$-band dynamics. Additionally, the rotational temperature of the molecules was also treated as a time-independent fit parameter.

The agreement of the analytical model with the $\tilde{\mathrm{A}} / \tilde{\mathrm{B}}-$ band dynamics (Fig. 8a) is good. All observed features, such as the non-exponential decay, the local minimum and the asymptotic value of the asymmetry parameter are well reproduced. However, the extracted photoionization parameters are difficult to interpret because the underlying vibronic dynamics in the $\tilde{\mathrm{A}} / \tilde{\mathrm{B}}$ band is not taken into account in this simple analytical model. This 
Table I. Rotational constants used in calculating the timedependent axis-distribution moments in Eq. (5).

\begin{tabular}{ccc}
\hline Constant & Rydberg state $\tilde{\mathrm{A}} / \tilde{\mathrm{B}}$-manifold \\
\hline \hline $\mathrm{A} / \mathrm{cm}^{-1}$ & 2.995 & 1.3288 \\
$\mathrm{~B} / \mathrm{cm}^{-1}$ & 0.302 & 0.3509 \\
$\mathrm{C} / \mathrm{cm}^{-1}$ & 0.274 & 0.2686 \\
$(\mathrm{~B}+\mathrm{C}) / 2 / \mathrm{cm}^{-1}$ & 0.2880 & 0.3098 \\
\hline
\end{tabular}

fact is also most likely to be responsible for the unexpectedly high rotational temperature of $T_{\text {rot }} \approx 766 \mathrm{~K}$ that was obtained from the fitting procedure. Using the rotational constants of either the $\tilde{B}$ or that of the $\tilde{A}$ state leads to similarly high rotational temperatures.

Turning to the $\tilde{\mathrm{F}}$-band dynamics (Fig. 8b), we find that the simple model fails to reproduce the observed time dependence of the asymmetry parameter. Although the best fit (red line) provides a more realistic rotational temperature of $T_{\text {rot }} \approx 146 \mathrm{~K}$, it clearly overestimates the initial decay rate until a delay of $0.2 \mathrm{ps}$, underestimates the rate between 0.2 and $0.3 \mathrm{ps}$, suggests the existence of a local minimum around $0.55 \mathrm{ps}$, which is not observed experimentally, and a non-zero asymptotic value. This significant disagreement is qualitatively consistent with the assumption that the $\tilde{\mathrm{F}}$-band signal is suppressed by the wavepacket leaving the ionization window, e.g. because of dissociative dynamics.

The comparison of the observed time-dependent anisotropies with the simple analytical model from the literature $^{47}$ thus shows that the observed time-dependent photoelectron angular distributions are not consistent with the assumptions of either time-independent parameters $B_{l, 2 i, q}(t)$ describing photoionization and/or the simple rigid-molecule assumption used in the calculation of the time-dependent axis-distribution moments. Therefore, more advanced theoretical work is required to fully account for the observed anisotropy parameters and to fully interpret the observed time-dependent photoelectron angular distributions.

\section{CONCLUSION}

In this work, we have measured time- and angleresolved photoelectron spectra of $\mathrm{SO}_{2}$. We have used femtosecond pulses centered at 133 and $266 \mathrm{~nm}$, allowing us to study dynamics following photo-excitation to the $\tilde{\mathrm{A}} / \tilde{\mathrm{B}}$ and $\tilde{\mathrm{F}}$ bands. In the case of the $\tilde{\mathrm{A}} / \tilde{\mathrm{B}}$ band prepared by the 266-nm pulse, no discernible temporal modulation was observed, which indicates that neither the photoion, nor the photoelectron signals are sensitive to the internalconversion dynamics. Excitation by the 133-nm pulse allowed for the first photoelectron study of the $\tilde{\mathrm{F}}$ band. In this case, we have observed a rapid decay with a time constant of $\sim 80 \mathrm{fs}$ in both photoion and photoelectron yields. The presented result are consistent with excitation around $9.3 \mathrm{eV}$ accessing an s-type Rydberg state, in which the photoexcited wave packet rapidly leaves the configuration space from where ionization takes place. Given the large excitation energy, dissociative dynamics is most likely. Finally, time-dependent asymmetry parameters have been measured and compared to a simple analytical model. This comparison confirms the observation of a time-dependent rotational anisotropy in the case of the $\tilde{\mathrm{A}} / \tilde{\mathrm{B}}$-band dynamics, which however requires the assumption of an unrealistically high rotational temperature. This indicates that electronic and/or vibrational dynamics also contribute to the observed variations of the asymmetry parameters. In the case of the $\tilde{\mathrm{F}}$-band dynamics, the asymmetry parameter also shows dynamics that initially resemble that of rotational anisotropy, however followed by a rapid decay to zero as the photoexcited wave packet leaves the configuration space from where single-photon ionization by a $266-\mathrm{nm}$ photon can take place.

Overall, these results demonstrate the potential of photoelectron imaging based on low-order harmonic generation. We have shown that femtosecond VUV pulses from such sources can be used to record dynamics taking place in low-lying electronic states of molecules, while avoiding ionization of unexcited molecules. Additionally, these sources enable the observation of Rydberg-state dynamics under the well-controlled conditions of single-photon excitation. Combined with photoelectron imaging, such sources have the potential of providing substantial new insight into coupled electronic and nuclear dynamics that were previously very challenging to access.

\section{Acknowledgement}

We thank Paul Hockett, Oliver Schalk, Horst Köppel, Camille Lévêque and Richard Taïeb for helpful discussions. This work has been supported by an ERC starting grant (contract no. 307270-ATTOSCOPE), and the Swiss National Science Foundation under project no. $200021 \_159875$.

\section{REFERENCES}

${ }^{1}$ A. T. J. B. Eppink and D. H. Parker, Rev. Sci. Instrum. 68, 3477 (1997).

${ }^{2}$ C. Bordas, F. Paulig, H. Helm, and D. L. Huestis, Rev. Sci. Instrum. 67, 2257 (1996).

${ }^{3}$ C. Vallance, Philosophical Transactions of the Royal Society A: Mathematical, Physical and Engineering Sciences 362, 2591 (2004).

${ }^{4} \mathrm{~T}$. Seideman, Annual Review of Physical Chemistry 53, 41 (2002).

${ }^{5}$ A. Stolow, A. E. Bragg, and D. M. Neumark, Chemical Reviews 104, 1719 (2004).

${ }^{6}$ K. L. Reid, Annual Review of Physical Chemistry (2003).

${ }^{7}$ T. Suzuki, Annu. Rev. Phys. Chem. 57, 555 (2006).

${ }^{8}$ T. Horio, T. Fuji, Y.-I. Suzuki, and T. Suzuki, J. Am. Chem. Soc. 131, 10392 (2009).

${ }^{9}$ T. Popmintchev et al., Science 336, 1287 (2012).

${ }^{10}$ Y. Pertot et al., Science (2017), doi:10.1126/science.aah6114. 
${ }^{11}$ F. Frassetto et al., Opt. Express 19, 19169 (2011).

${ }^{12}$ A. von Conta, M. Huppert, and H. J. Wörner, Review of Scientific Instruments $\mathbf{8 7}$ (2016)

${ }^{13}$ L. Nugent-Glandorf et al., Phys. Rev. Lett. 87, 193002 (2001).

${ }^{14}$ L. Nugent-Glandorf, M. Scheer, D. A. Samuels, V. M. Bierbaum, and S. R. Leone, J. Chem. Phys. 117, 6108 (2002).

${ }^{15}$ A. Makida et al., J. Phys. Chem. Lett. 5, 1760 (2014).

${ }^{16}$ H. J. Wörner, J. B. Bertrand, D. V. Kartashov, P. B. Corkum, and D. M. Villeneuve, Nature 466, 604 (2010).

${ }^{17}$ H. J. Wörner, J. B. Bertrand, P. B. Corkum, and D. M. Villeneuve, Phys. Rev. Lett. 105, 103002 (2010).

${ }^{18}$ H. J. Wörner et al., Science 334, 208 (2011).

${ }^{19}$ P. M. Kraus and H. J. Wörner, Chemical Physics 414, 32 (2013).

${ }^{20}$ A. Tehlar and H. J. Wörner, Molecular Physics 111, 2057 (2013).

${ }^{21} \mathrm{~T}$. Horio et al., The Journal of Chemical Physics 145 (2016).

${ }^{22}$ I. Wilkinson et al., The Journal of Chemical Physics 140, 204301 (2014).

${ }^{23}$ H. Müller and H. Köppel, Chem. Phys. 183, 107 (1994).

${ }^{24}$ C. Lévêque, A. Komainda, R. Taïeb, and H. Köppel, The Journal of Chemical Physics 138 (2013).

${ }^{25}$ C. Xie, X. Hu, L. Zhou, D. Xie, and H. Guo, The Journal of Chemical Physics 139, 014305 (2013).

${ }^{26}$ C. Lévêque, R. Taïeb, and H. Köppel, The Journal of Chemical Physics 140, 091101 (2014).

${ }^{27}$ C. Lévêque, H. Köppel, and R. Taïeb, The Journal of Chemical Physics 140, 204303 (2014).

${ }^{28}$ D.-D. Zhang et al., Chinese Physics Letters 28, 033301 (2011).

${ }^{29}$ E. S. Wisniewski and A. W. J. Castleman, The Journal of Physical Chemistry A 106, 10843 (2002).

${ }^{30}$ B. Xue, Y. Chen, and H.-L. Dai, The Journal of Chemical Physics 112, 2210 (2000).
${ }^{31}$ L. Wang, Y. T. Lee, and D. A. Shirley, The Journal of Chemical Physics 87, 2489 (1987).

${ }^{32}$ P. J. Singh, A. Shastri, R. D. Souza, S. B. Rao, and B. Jagatap, Journal of Quantitative Spectroscopy \& Radiative Transfer 113, 267 (2012).

${ }^{33}$ K. L. Knappenberger and A. W. Castleman, J. Phys. Chem. A 108, 9 (2004).

${ }^{34}$ H. Katagiri et al., Journal of Molecular Structure 413-414, 589 (1997).

${ }^{35}$ T. Sako, A. Hishikawa, and K. Yamanouchi, Chemical Physics Letters 294, 571 (1998).

${ }^{36}$ K. Yamanouchi, M. Okunishi, Y. Endo, and S. Tsuchiya, Journal of Molecular Structure 352-353, 541 (1995).

${ }^{37}$ M. H. Palmer, D. A. Shaw, and M. F. Guest, Molecular Physics 103, 1183 (2005).

${ }^{38} \mathrm{C}$. Lévêque, private communication (2014).

${ }^{39}$ S. G. Walt et al., J. Phys. Chem. A 119, 11772 (2015).

${ }^{40} \mathrm{~S}$. G. Walt, Imaging Electronic Structure and Dynamics of Molecules through Strong-Field Ionization, Rescattering and Holography, PhD thesis, ETH, 2015.

${ }^{41}$ G. A. Garcia, L. Nahon, and I. Powis, Rev. Sci. Instrum. 75, 4989 (2004).

${ }^{42}$ C. A. F. Johnson, S. D. Kelly, and J. E. Parker, J. Chem. Soc., Faraday Trans. 2 83, 411 (1987).

${ }^{43}$ D. Holland et al., Chemical Physics 188, 317 (1994).

${ }^{44}$ S. Pedersen and A. H. Zewail, Molecular Physics 89, 1455 (1996).

${ }^{45}$ T. Suzuki, L. Wang, and M. Tsubouchi, J. Phys. Chem. A 108, 5764 (2004)

${ }^{46}$ M. Tsubouchi, B. J. Whitaker, and T. Suzuki, J. Phys. Chem. A 108, 6823 (2004).

${ }^{47}$ O. Schalk and P. Hockett, Chemical Physics Letters 517, 237 (2011). 